\title{
ON UNIVERSAL CHARACTER OF THE PRIMITIVE LOGIC
}

\author{
KATUZI ONO \\ To the memory of TADASI NAKayama
}

The PRIMITIVE LOGIC LO introduced in my former work ${ }^{1)}$ is a logic having only two logical constants IMPLICATION $\rightarrow$ and UNIVERSAL QUANTIFICATION ( ) with their usual inference rules which are admitted even in the INTUITIONISTIC PREDICATE LOGIC LJ. LO is really a very simple logic, maybe the simplest possible logic as one can imagine, but it is very important because of its universal character. In fact, popular logics such as the LOWER CLASSICAL PREDICATE LOGIC LK, the INTUITIONISTIC PREDICATE LOGIC LJ, the MINIMAL PREDICATE LOGIC $\mathbf{L M}^{2)}$, etc. can be faithfully interpreted in it. Speaking frankly, I am further expecting that all the important logics would be interpreted faithfully in it and would disclose their intrinsic characteristics by being interpreted in it. Main purpose of this paper is to show the universal character of the primitive logic LO by pointing out that a series of typical logics are faithfully interpretable in LO.

The lower classical predicate logic $\mathbf{L K}$ and the intuitionistic predicate logic LJ may be the most popular logics. $\mathbf{L K}$ is a logic stronger than $\mathbf{L J}$ in the sense that all the propositions provable in $\mathbf{L J}$ are surely provable in LK but propositions provable in $\mathbf{L K}$ are not always provable in LJ. However, we can not expect that any pair of logics are always in such situation. For example, we can really reason in a logic in which tertium non datur holds but not the principle that contradiction implies everything. Let us denote this logic by LD according to Curry ${ }^{3)}$. Then, propositions provable in $\mathbf{L J}$ are not always prov-

Received June 23, 1965.

1) See ONO [1]. The PRIMITIVE LOGIC LO is called the PRIMITIVE SYSTEM OF POSITIVE LOGIC in ONO [1]. LO is a notation introduced in the present paper.

2) See JOHANSSON [1]. See also ONO [1], especially, the system O-PLM.

3) See CURRY [1], [2]. See also $(2.2 \mathrm{R})$. It is really an interesting task to interpret faithfully $\mathbf{L D}$ in $\mathbf{L O}$. 
able in $\mathrm{LD}$ and also propositions provable in $\mathrm{LD}$ are not always provable in LJ. It may sound rather strange that the same person reasons occasionally in $\mathbf{L J}$ and occasionally in LD. In reality, however, we can really reason in such manner at our will.

I can not imagine such situation unless something universal is hidden behind the background of the various logics and they can be regarded as various expressions of the same thing just as the same thing can be expressed differently in various languages. If so, it must be our most interesting task to disclose the something universal behind the background.

Indeed, the classical logic can be regarded as the logic for the world of possibility, i.e. for propositions whose NEGATIONS can be denied. So, the classical logic can be formally interpreted in the intuitionistic logic as the logic for double-negative propositions $\neg \neg \mathfrak{P}^{4}$ ). (In the intuitionistic logic, any negative proposition can be regarded as a double-negative proposition.) Naturally, some logical constants such as $\vee$ or $(\exists)$ must be suitably interpreted.

The way of reduction can be prolonged still further. The classical logic LK can be reduced even to the primitive logic LO. In LO, we have no NEGATION notion, but NEGATION notion can be interpreted easily, assuming that there is a fixed contradictory proposition $\wedge^{5)}$ which implies everything. Naturally, I would not assume such a thing from the beginning. However, even if we give up the proper meaning of $\wedge$ in $\mathbf{L K}$ and regard it just a SYMBOL FOR A FIXED PROPOSITION, we have a logic somewhat similar to LK by regarding $\mathfrak{A} \rightarrow \wedge$ as $\rightarrow \mathfrak{A}$, or shortly $\rightarrow \wedge$ as $\rightarrow$. Main difference is that $\wedge$ does not imply everything. So, we might take a step forward by taking $\wedge$ just a NEW SYMBOL and by dealing with only such propositions that are deducible from $\wedge$, i.e. propositions expressible in the form $\mathfrak{A} \rightarrow \wedge$.

But, what is a NEW SYMBOL? To speak more exactly on interpretation of a logic in another logic, we had better establish a convention to use the words LOGIC and LOGICAL SYSTEM in different meanings. When I speak of LOGIC, I do not mind whatever elementary relations may be used, I deal only with a finite set of elementary logical constants (I will call elementary

4) See GLIVENKO [1]. See also KURODA [1] and ONO [1].

5) In this paper, I employ occasionally the Gentzen's symbol $\wedge$ for CONTRADICTION (See GENTZEN [1].) instead of the logical constant NEGATION $\rightarrow$. $\wedge$ is a propositional çonstant and can be regarded as a primitive notion (0-ary relation symbol), if one will, 
logical constants of the logic L L-CONSTANTS, hereafter.) and inference rules with respect to its logical constants. But when I speak of LOGICAL SYSTEM, I presuppose a certain finite set of primitive notions for each system together with the logic it stands on. Accordingly, any logical system has its own set of elementary relations called its VOCABULARY and its own logic. Formal systems usually assume further something proper to their respective vocabularies, but $\mathrm{I}$ assume nothing of this kind for logical systems $\mathbf{s}^{6}$.

Accordingly, in any logical system, we can not find out any difference in properties of any pair of elementary relations of the same number of variables in its vocabulary. For example, in formal theories such as set theories, $\in$ and $=$ are two distinct elementary relations having different properties, the one satisfies $x=x$ and the other does not satisfy $x \in x$. However, this distinction comes from assumptions proper to $\in$ and $=$, i.e. from assumptions of the formal theories, but not from their logical systems. We can consider a logical system having the pair of distinct elementary relations $\in$ and $=$, but if a proposition $\mathfrak{A}$ is provable with respect to $\in$ (e.g. $x \in y \rightarrow x \in y$ ), then it must be also provable in the same logical system with respect to $=($ i.e. $x=y \rightarrow x=y$ ). In the logical system, $\in$ and $=$ are merely two distinct binary relations.

Any logical system standing on a logic $\mathbf{L}$ is called an L-SYSTEM, e.g. LOsystem, LK-system, etc. The L-system having the vocabulary $\alpha$ is denoted by $\mathbf{L}(\alpha)$, e.g. $\mathbf{L O}(\alpha), \mathbf{L K}(\alpha)$, etc. ${ }^{7}$ NEW RELATION SYMBOLS (including propositions regarded as 0 -ary relations) can be given for any logical system, but not for logic itself. Namely, any relation symbol $S$ lying outside of the vocabulary of a logical system can be called a NEW SYMBOL for the logical system. Accordingly, our previous surmise can be stated as follows: LK $(\alpha)$ would be faithfully interpreted in $\operatorname{LO}(\{F\} \cup \alpha)$ assuming that $F$ does not belong to $\alpha$, or I would rather say that $\mathbf{L K}(\alpha)$ is embedded into $\operatorname{LO}(\{F\} \cup \alpha)$. I will introduce in (1) the notion FAITHFUL INTERPRETATION of a logic $\mathrm{L}$ in another logic $\mathbf{L}^{*}$ after defining EMBEDDING of a logical system $\mathbf{L}(\alpha)$ into another logical system $\mathbf{L}^{*}\left(\boldsymbol{\alpha}^{*}\right)$.

Now, by introducing a new symbol $F$ for any LK-system, we can make an

6) See Foot-note 5 ).

7) In this paper, I assume that the vocabulary $\alpha$ of any logical system is a finite set of elementary relations. 
exact copy of the LK-system in a certain LJ-system. Furthermore, we can continue the reduction even to Lo. In fact, $\rightarrow F$ can be taken as the copy of $\rightarrow$, so the $F$.CLOSURE $\mathfrak{U}^{F}$ of $\mathfrak{A}$ defined as standing for $(\mathfrak{H} \rightarrow F) \rightarrow F$ is the copy of double negation of $\mathfrak{A}$. Hence, the characteristic property of double negative proposition $\mathfrak{A}$ can be described as $F$-CLOSED which means that $\mathfrak{A}^{F}$ implies $\mathfrak{H}$. Since all the logical constants of $\mathbf{L K}$ can be defined by means of $\rightarrow,()$, and $\rightarrow$, we may make the copies of all the logical constants of $\mathbf{L K}$ in terms of $\rightarrow$, $($ ), and the new symbol $F$. Indeed, any LK-system $\mathbf{L K}(\alpha)$ can be embedded into an LO-system $\operatorname{LO}(\{F\} \cup \alpha)$ assuming that $F$ does not belong to $\alpha$.

It must be a matter of course of our free thinking to seek for a similar interpretation of $\mathbf{L J}$ in LO. However, we are confronted with an essential difficulty in defining copies of logical constants of $\mathbf{L J}$ other than $\rightarrow$ and ( ) in LO. For example, we can not define $\mathfrak{A} \wedge \mathfrak{B}$ by $\rightarrow(\mathfrak{A} \rightarrow \neg \mathfrak{B})$ or by $(\mathfrak{A} \rightarrow(\mathfrak{B} \rightarrow F)) \rightarrow$ $F$, because $\mathfrak{A} \wedge \mathfrak{B}$ should not be equivalent to $\rightarrow(\mathfrak{A} \rightarrow \rightarrow \mathfrak{B})$ in general in $\mathbf{L J}$. At present, I am rather of the opinion that the copies of logical constants $\wedge$, $\vee$, and $(\exists$ ) of LJ can be defined only by making use of predicate-logical device in LO.

The leading idea of the method I am going to introduce here for this purpose is that the conditions of the form $(\xi)((\mathfrak{A} \rightarrow \mathfrak{N}(\xi)) \rightarrow \mathfrak{N}(\xi))$ is much stronger than and quite different from the usual double-negative form $\rightarrow \rightarrow \mathfrak{A}$ or its interpretation $(\mathfrak{A} \rightarrow \mathfrak{F}) \rightarrow \mathfrak{F}$ for any fixed proposition $\widetilde{F}$, if the relation $\mathfrak{R}$ is able to express sufficiently large class of propositions in the form $\Re(\xi)$. (I denote by small greek letters $\xi$ series of variables such as $x_{1}, \ldots, x_{n}$. If $\xi$ denotes $x_{1}, \ldots, x_{n},(\xi) \mathfrak{A}(\xi)$ naturally denotes $\left.\left(x_{1}\right) \cdots\left(x_{n}\right) \mathfrak{U}\left(x_{1}, \ldots, x_{n}\right).\right)$ In LJ, we can not expect that $\mathfrak{A}$ is deducible from $(\mathfrak{A} \rightarrow \mathfrak{F}) \rightarrow \mathfrak{F}$ for any fixed $\mathfrak{F}$. However, we can really find out even in LJ such $\mathfrak{F}$ for any $\mathfrak{F}$ that $(\mathfrak{A} \rightarrow \mathfrak{F}) \rightarrow \mathfrak{F}$ implies $\mathfrak{A}$. (Take for example $\mathfrak{U}$ for $\mathfrak{F}$.) If the class of propositions $\mathfrak{R}(\xi)$ is wide enough, the class of such propositions that $(\xi)((\mathfrak{U} \rightarrow \mathfrak{R}(\xi)) \rightarrow \mathfrak{N}(\xi))$ implies $\mathfrak{A}$ can be taken as the domain of propositions of LJ. I will call the proposition $(\xi)((\mathfrak{A} \rightarrow \mathfrak{R}(\xi)) \rightarrow \mathfrak{R}(\xi))$ the $\mathfrak{R}$-CLOSURE of $\mathfrak{H}$ and denote it by $\mathfrak{U}^{\Re}$. $\mathfrak{A}$ is called $\Re$-CLOSED if and only if $\mathfrak{H}^{\Re}$ implies $\mathfrak{H}$. Evidently, the notion $\mathfrak{R}$-CLOSED PROPOSITION is a generalization of the notion $\widetilde{f}$-CLOSED PROPOSITION regarding $\mathfrak{F}$ as a 0 -ary relation. The class of $\mathfrak{R}$-closed propositions is closed with respect to the logical constants $\rightarrow$ and ( ). 
In reality, it would be impossible to confirm for any relation $\Re$ that the class of propositions expressible in the form $\Re(\sigma)$ is wide enough for our purpose. Hawever, if we take up a new relation symbol $R$, it would be also impossible to confirm that the class of propositions expressible in the form $R(\sigma)$ is not wide enough for our purpose. The assertion that a proposition $\mathfrak{A}$ containing a new relation symbol $R$ is provable is really a very strong assertion for $\mathfrak{H}$. $\mathfrak{A}$ would not be logically provable unless $\mathfrak{A}$ is provable in the imaginary case that every proposition is expressible in the form $R(\sigma)$.

In this connection, I would like to show another example for usage of new symbols. In LO, we have no NEGATION, and we can not confirm that there is surely a fixed contradictory proposition. However, we are also unable to confirm that there is no contradictory proposition at all. Accordingly, any proposition $\mathfrak{A}$ containing a new proposition symbol $F$ would not be provable unless $\mathfrak{A}$ is provable in the imaginary case that $F$ is a contradiction. This would show a merit of our usage of NEW proposition- or relation-symbols.

With respect to any relation $\Re$, we can define $\mathfrak{R}$-CONJUNCTION $\underset{\Re}{\wedge}, \mathfrak{R}$ DISJUNCTION $\underset{\Re}{\vee}$, and $\Re$-EXISTENTIAL QUANTIFICATION $(\underset{\Re}{\exists})$ by the following :

$$
\begin{aligned}
& \mathfrak{U} \wedge \mathfrak{\Re} \mathfrak{B} \equiv(\xi)((\mathfrak{A} \rightarrow(\mathfrak{B} \rightarrow \mathfrak{N}(\xi))) \rightarrow \mathfrak{R}(\xi)), \\
& \mathfrak{A} \vee \mathfrak{B} \equiv(\xi)((\mathfrak{A} \rightarrow \mathfrak{R}(\xi)) \rightarrow((\mathfrak{B} \rightarrow \mathfrak{R}(\xi)) \rightarrow \mathfrak{R}(\xi))), \\
& (\underset{\mathfrak{R}}{\exists} u) \mathfrak{A}(u) \equiv(\xi)((\boldsymbol{u})(\mathfrak{A}(\boldsymbol{u}) \rightarrow \mathfrak{N}(\xi)) \rightarrow \mathfrak{R}(\xi))^{8)} \text {. }
\end{aligned}
$$

According to the above definitions, $\mathfrak{Y} \underset{\mathfrak{R}}{\wedge} \mathfrak{B}, \mathfrak{A} \underset{\mathfrak{R}}{\vee} \mathfrak{B}$, and $\underset{\mathfrak{M}}{\exists} u) \mathfrak{H}(\boldsymbol{u})$ are all $\mathfrak{A}$-closed for any $\mathfrak{A}, \mathfrak{B}$, and $\mathfrak{Y}(p)$, and $\underset{\Re}{\wedge}, \underset{\Re}{\vee}$, and $(\underset{\Re}{\exists})$ together with the LO-constants $\rightarrow$ and ( ) satisfy in LO the usual inference rules for CONJUNCTION, DISJUNCTION, EXISTENTIAL QUANTIFICATION, IMPLICATION, and UNIVERSAL QUANTIFICATION of the POSITIVE LOGIC $\mathbf{L P}^{9)}$, i.e. the intuitionistic predicate logic without NEGATION notion, for $\Re$-closed propositions. If we take up for any LP-system $\operatorname{LP}(\alpha)$ a new $n$-ary relation symbol $R$ (NEW in the sense that $R$ does not belong to $\alpha$ ) for a sufficiently large $n$, then $\rightarrow$, ()$, \wedge_{R}^{\wedge}, \underset{R}{\vee}$, and $(\exists)$ can be taken as the copies of $\rightarrow,(), \wedge, \vee$, and $(\exists)$

8) Propositions of the form $\mathfrak{A} \equiv \mathbb{B}$ denote that $\mathfrak{A}$ stands for $\mathfrak{B}$, or in other words, $\mathfrak{A}$ is defined by $\mathfrak{B}$.

9) See HILBERT and BERNAYS [1], LORENZEN [1], CURRY [1], [2], and ONO [1]. 
of $\operatorname{LP}(\alpha)$ in $\operatorname{LO}(\{R\} \cup \alpha)$, respectively, regarding the $R$-closure of every elementary proposition of $\mathbf{L P}(\alpha)$ as the copy of the elementary proposition. The way from $\mathbf{L P}$ to $\mathbf{L J}$ is rather easy. (See $(\mathbf{2 . 9} \mathbf{T})^{10)}$.) We can thus interpret the intuitionistic predicate logic $\mathbf{L J}$ in the primitive logic LO.

I can further show in the present paper that any one of the following logics can be faithfully interpreted in LO.

LP: Positive predicate logic without NEGATION.

LQ: Positive predicate logic fortified by Peirce's rule that $(\mathfrak{A} \rightarrow \mathfrak{B}) \rightarrow \mathfrak{A}$ implies $\mathfrak{A}^{11}$.

LM: Minimal predicate logic.

LN : Minimal predicate logic fortified by Peirce's rule.

LJ : Intuitionistic predicate logic.

LK: Lower classical predicate logic.

These logics are defined by their inference rules with respect to their logical constants. I will give a sketch of these logics in (2). LP, LM, and LJ are called J-SERIES LOGICS, and LQ, LN, and LK are called K-SERIES LOGICS.

Any logic can be characterized by the class of propositions provable in it. Accordingly, two logics can be duly called EQUIVALENT if and only if the respective classes of propositions provable in them are the same. (If every proposition provable in a logic $\mathbf{L}$ is also provable in another $\operatorname{logic} \mathbf{L}^{*}, \mathbf{L}$ is called WEAKER than $\mathbf{L}^{*}$.) If either of two logics can be faithfully interpreted in the other, the two logics can be duly called INTERPRETABLY EQUIVALENT. The logics LO, LP, LM, and LJ are not equivalent to each other, but they are interpretably equivalent to each other as shown later.

Any propsoition (or any relation) expressible without employing any logical constants other than $\rightarrow$ and ( ) is called an O-PROPOSITION (O-RELATION), and any proposition (relation) expressible without employing any logical constants other than $\rightarrow,(), \wedge, \vee$, and $(\exists)$ is called a P-PROPOSITION ( $P$ RELATION). J- and $\mathbf{K}$-series logics are so defined that any P-proposition

10) The symbols $\mathbf{D}, \mathbf{T}$, and $\mathbf{R}$ in numberings of the forms $(\mathbf{m}, \mathbf{n D}),(\mathbf{m}, \mathbf{n T})$, and $(\mathbf{m}, \mathbf{n R})$ denote that $(\mathbf{m}, \mathbf{n D}),(\mathbf{m}, \mathbf{n} \mathbf{T})$, and $(\mathbf{m}, \mathbf{n R})$ are a DEFINITION, a THEOREM, and a REMARK, respectively.

11) See PEIRCE [1] (fifth icon). As for detailed comparison of tertium non datur and Peirce's rule, see (2.2 R). As for $\mathbf{L P}$ and $\mathbf{L Q}$, see LORENZEN [1] (Positiver Logikkalkül and Positiver Logikkalkül nach Hinzunahme der PEIRCEschen Aussage). 
provable in a $\mathbf{J}$-series logic is also provable in any other $\mathbf{J}$-series logic and that any $\mathbf{P}$-proposition provable in a $\mathbf{K}$-series logic is also provable in any other $\mathrm{K}$-series logic. (See $(2.6 \mathrm{~T})$ and $(2.7 \mathrm{~T})$.) Mutual interpretable equivalence of $\mathbf{J}$-series logics as well as of $\mathbf{K}$-series logics is shown in (2).

In (3), I describe some fundamental properties, especially of $\Re$-closure for any relation $\Re$. Fundamental properties of $\Re$-conjunction $\wedge_{\Re}$, $\Re$-disjunction $\underset{\Re}{\vee}$, and $\Re$-existential quantification $(\underset{\Re}{\exists})$ are described in (4).

By making use of these notions, I will show in (5) that LP and LQ can be faithfully interpreted in LO. It is rather easy to prove that the copy $\mathfrak{I}^{*}$ of $\mathfrak{A}$ is provable in the corresponding LO-system for any proposition $\mathfrak{A}$ provable in the original LP. or LQ-system. It is also easy to see conversely for LQ that $\mathfrak{A}$ is provable in any $\mathbf{L Q}$-system as far as $\mathfrak{A}^{*}$ is provable in the corresponding LO-system.

To illustrate how to prove the same assertion for LP, I begin with the following speculations: Let us at first consider an LP-system having only one $n$-ary elementary relation $A$. Let us denote by $\mathfrak{A}[R]$ the copy of $\mathfrak{A}$ with respect to the new relation symbol $R$. If $\mathfrak{A}[R]$ is provable for any $\boldsymbol{n}$-ary relation symbol $R$ in $\mathbf{L O}(\{R, A\})$, then $\mathfrak{A}[A]$ must be provable in $\mathbf{L O}(\{A\})$. Hence, $\mathfrak{A}[A]$ is also provable in $\operatorname{LP}(\{A\})$. On the other hand, every elementary formula $A\left(p_{1}, \ldots, p_{n}\right)$ would be $A$-closed, and moreover, $\underset{A}{\wedge}, \underset{A}{\vee}$, and $\left.\underset{A}{\exists}\right)$ would show no distinction from $\wedge, \vee$, and $(\exists$ ) for $A$-closed formulas, respectively. So, $\mathfrak{A}$ itself must be provable in $\operatorname{LP}(\{A\})$.

Next, let us consider the positive proposition logic. If we associate to each elementary proposition $A_{i}$ a free variable $a_{i}$ and take up a predicate symbol $P$ regarding it as a 1 -ary relation, then any proposition $\mathfrak{A}$ is provable in LP if and only if the proposition $\mathfrak{H}^{*}$ obtained on replacing all the elementary proposition $A_{i}$ by $P\left(a_{i}\right)$ is provable in LP. Now, $\mathfrak{A}^{*}$ is a proposition of the logical system $\mathbf{L P}(\{P\})$ having only one elementary relation $P$, so the positive proposition logic LP can be embedded in the logical system $\mathbf{L O}(\{P\})$.

The leading idea of my proof for the general case is just a modification of these speculations. I will illustrate it taking up again LP as an example logic. Broadly speaking, for any LP-system $\mathbf{L P}(\alpha)$, we have only to introduce a system having only one elementary relation which behave similarly as $\operatorname{LP}(\alpha)$. Namely, let the vocabulary $\alpha$ be the set $\left\{A_{1}, \ldots, A_{k}\right\}, A_{i}$ be an $n(i)$-ary 
relation for every $i$, and $n$ be a number no less than any one of $n(i)$ 's. Further, let $P$ be an $(n+1)$-ary relation symbol not belonging to the vocabulary $\alpha$, and let $a_{1}, \ldots, a_{k}$, and $d$ be mutually distinct free variables which do not occur in $\mathfrak{A}$. The variables $a_{i}$ serve for expressing $A_{i}\left(p_{1}, \ldots, p_{n(i)}\right)$ in the form $P\left(a_{i}, p_{1}, \ldots, p_{n(i)}, d, \ldots, d\right), d$ being a dummy symbol.

If $\mathfrak{A}[R]$ is provable in $\operatorname{LO}(\{R\} \cup \alpha)$ for an $(n+1)$-ary relation symbol $R$, the proposition $\mathfrak{A}[R]^{*}$ obtaind on replacing all the elementary formulas of the form $A_{i}\left(p_{1}, \ldots, p_{n(i)}\right)$ in $\mathfrak{2}[R]$ by $P(a_{i}, p_{1}, \ldots, p_{n(i)}, \underbrace{d, \ldots, d}_{n-n(i)})$ would be provable in $\mathbf{L O}(\{R\})$. Accordingly, $\mathfrak{A}^{*}[P]$ would be provable in $\mathbf{L O}(\{P\})$, so $\mathfrak{A}^{*}[P]$ would be provable in $\mathbf{L P}(\{P\})$. Because every proposition $P\left(a_{i}, p_{1}, \ldots, p_{n}\right)$ is $P$.closed, and moreover, $\wedge_{P}, \underset{P}{\vee}$, and $(\underset{P}{\exists})$ would show no distinction from $\wedge, \vee$, and $(\exists)$ for $P$-closed propositions, respectively, so the proposition $\mathfrak{H}^{*}$ obtained on replacing every elementary formula of the form $A_{i}\left(p_{1}, \ldots, p_{n(i)}\right)$ in $\mathfrak{A}$ by $P\left(a_{i}, p_{1}, \ldots, p_{n(i,}, d, \ldots, d\right)$ would be also provable in $\operatorname{LP}(\{P\})$. This leads to the conclusion that $\mathfrak{A}$ itself is provable in $\operatorname{LP}(\alpha)^{13)}$.

Proofs in this paper are described rather informally. I am planning to write a book which contains the whole theory of the present work described formally in details.

(1) Embedding of a logical system in another logical system and faithful interpretation of a logic in another logic

For any logic L, we assume a finite set of L-constants, and for any Lsystem $\mathrm{L}(\alpha)$, we assume a finite vocabulary $\alpha$ of it. We call any proposition $\mathfrak{U}$ a PROPOSITION OF $\mathbf{L}(\alpha)$ if and only if $\mathfrak{A}$ is a proposition expressible in terms of the vocabulary $\alpha$ together with L-constants. By making use of any series of mutually distinct variables $p_{1}, \ldots, p_{s}$ which do not occur in the proposition $\mathfrak{U}\left(q_{1}, \ldots, q_{s}\right)$ of $\mathbf{L}(\alpha)$, an $s$-ary relation of $p_{1}, \ldots, p_{s}$ is defined by $\mathfrak{U}\left(p_{1}, \ldots, p_{s}\right)$, which is simply called an $s$-ARY RELATION OF $\mathbf{L}(\alpha)$ and simply denoted by $\mathfrak{A}$ if there is no fear of ambiguity.

For any logical system $\mathbf{L}(\alpha)$, we assume that the proposition obtained on replacing every $s$-ary elementary relation $R_{i}$ of any proposition $\mathfrak{A}$ provable in $\mathbf{L}(\alpha)$ by an $s$-ary relation $\Re_{i}^{*}$ of $\mathbf{L}\left(\alpha^{*}\right)$ is provable in $\mathbf{L}\left(\alpha^{*}\right)$.

12) The device adopted in this proof for denoting elementary propositions in a single standard type is closely connected with the method employed in my old work, ONO [2]. 
We can define also logical constants in logical systems. We need in the present paper only the following four kinds of logical constants :

OPERATORS for propositions which map every proposition ( $\boldsymbol{n}$-ary relation) to a proposition $(\boldsymbol{n}$-ary relation). (Examples. NEGATION $: \mathfrak{A} \longrightarrow \rightarrow \mathfrak{A} . \quad \mathfrak{R}$ CLOSURE : $\mathfrak{A} \longrightarrow \mathfrak{l}^{\mathfrak{R}}$.)

Binary CONNECTIVES which map every pair of propositions to a propusition. (Examples. IMPLICATION : $\mathfrak{A}, \mathfrak{B} \longrightarrow \mathfrak{A} \rightarrow \mathfrak{B}$. CONJUNCTION : $\mathfrak{A}, \mathfrak{B} \longrightarrow$ $\mathfrak{H} \wedge \mathfrak{B} . \quad \mathfrak{R}$-DISJUNCTION : $\mathfrak{A}, \mathfrak{B} \longrightarrow \mathfrak{A} \underset{\mathfrak{R}}{\vee \mathfrak{B} .)}$

OPERATORS for predicates which map every predicate $((n+1)$-ary relation) to a proposition ( $n$-ary relation). (Examples. UNIVERSAL QUANTIFICATION : predicate $\mathfrak{A} \longrightarrow$ proposition $(x) \mathfrak{A}(x)$. $\mathfrak{R}$-EXISTENTIAL QUANTIFICATION : predicate $\mathfrak{A} \longrightarrow$ proposition $(\underset{\mathfrak{K}}{\exists} \boldsymbol{x}) \mathfrak{U}(x)$.)

PROPOSITIONAL CONSTANTS. (Example. CONTRADICTION : ^.)

(1.1 D) Embedding of a logical system into another logical system. Let $\mathbf{L}(\alpha)$ and $\mathbf{L}^{*}\left(\alpha^{*}\right)$ be any pair of logical systems. Any word-for-word translation $\mathbf{E}$ of propositions of $\mathbf{L}(\alpha)$ into propositions of $\mathbf{L}^{*}\left(\alpha^{*}\right)$ is called an EMBEDDING of $\mathbf{L}(\alpha)$ into $\mathbf{L}^{*}\left(\alpha^{*}\right)$ if and only if the following holds: Any proposition $\mathfrak{U}$ of $\mathbf{L}(\alpha)$ is provable in $\mathbf{L}(\alpha)$ if and only if the word-for-word translation $\mathfrak{A}^{*}$ of $\mathfrak{A}$ by $\mathbf{E}$ is provable in $\mathbf{L}^{*}\left(\alpha^{*}\right)$. Evidently, any embedding $E$ translates every $\mathbf{L}$-constants $\lambda_{i}$ into a logical constants $\lambda_{i}^{*}$ (defined in $\mathbf{L}^{*}\left(\alpha^{*}\right)$ ) of the same kind as $\lambda_{i}$ and every elementary $n(j)$-ary relation $R_{j}$ in $\alpha$ into an $n(j)$-ary relation $\mathfrak{R}_{j}^{*}$ of $\mathbf{L}^{*}\left(\alpha^{*}\right)$. Conversely, $\mathbf{E}$ is characterized by the mapping

$$
\mathrm{E}: \quad \lambda_{i} \longrightarrow \lambda_{i}^{*}, \quad R_{j} \longrightarrow \Re^{*}
$$

for every $i$ and $j . \quad \lambda_{i}^{*}$ and $\Re_{j}^{*}$ are called the COPIES of $\lambda_{i}$ and $R_{i}$ by the embedding $E$, respectively.

(1.2 D) Any logic $\mathbf{L}$ is called a SUBLOGIC of another logic $\mathbf{L}^{*}$ if and only if every $\mathbf{L}$-constant is an $\mathbf{L}^{*}$-constant and any proposition expressible in $\mathbf{L}$ is provable in $\mathbf{L}$ if and only if it is provable in $\mathbf{L}^{*}$. If either of two logics is a sublogic of the other, they are called EQUIVALENT.

Any sublogic $\mathbf{L}$ of a logic $\mathbf{L}^{*}$ is a logic weaker than $\mathbf{L}^{*}$.

Any logical system $\mathbf{L}(\alpha)$ can be embedded into another logical system $\mathbf{L}^{*}\left(\alpha^{*}\right)$ if $\mathbf{L}$ is a sublogic of $\mathbf{L}^{*}$ and $\alpha$ is a subset of $\alpha^{*}$. 
(1.3 R) Embedding is transitive in the sense that any logical system $\mathbf{L}(\alpha)$ can be embedded into another logical system $\mathbf{L}^{*}\left(\alpha^{*}\right)$ if $\mathbf{L}(\alpha)$ can be embedded into a logical system $\mathbf{L}^{* *}\left(\alpha^{* *}\right)$ and $\mathbf{L}^{* *}\left(\boldsymbol{\alpha}^{* *}\right)$ can be embedded into $\mathbf{L}^{*}\left(\alpha^{*}\right)$.

(1.4 D) Faithful interpretation of a logic in another logic. Let $\mathbf{L}$ and $\mathbf{L}^{*}$ be a pair of logics. Any rule $I$ which associates to every vocabulary $\alpha$ a vocabulary $\alpha^{*}$ and an embedding $\mathbf{E}$ of $\mathbf{L}(\alpha)$ into $\mathbf{L}^{*}\left(\alpha^{*}\right)$ is called a FAITHFUL INTERPRETATION of $\mathbf{L}$ in $\mathbf{L}^{*}$. I say that $\mathbf{L}$ is faithfully interpreted in $\mathbf{L}^{*}$ if a faithful interperetation of $\mathbf{L}$ in $\mathbf{L}^{*}$ is given. Broadly speaking, $\mathbf{L}$ can be faithfully interpreted in $\mathbf{L}^{*}$ if and only if every $\mathbf{L}$-system can be embedded into an $\mathbf{L}^{*}$-system.

If either of two logics can be faithfully interpreted in the other, they are called INTERPRETABLY EQUIVALENT.

(1.5 R) Any sublogic $\mathbf{L}$ of a $\operatorname{logic} \mathbf{L}^{*}$ can be faithfully interpreted in $\mathbf{L}^{*}$. Two logics are interpretably equivalent if they are equivalent.

(1.6 R) Faithful interpretation is transitive in the sense that any logic $\mathbf{L}$ can be faithfully interpreted in another $\operatorname{logic} \mathbf{L}^{*}$ if $\mathbf{L}$ is faithfully interpreted in the third logic $\mathbf{L}^{* *}$ and $\mathbf{L}^{* *}$ is faithfully interpreted in $\mathbf{L}^{*}$.

Interpretable equivalence of logics is surely reflexive, symmetric, and transitive.

(1.7 R) It should be remarked that a faithful interpretation $I$ of $\mathbf{L}$ in $\mathbf{L}^{*}$ do not always associate to the same $\mathbf{L}$-constant the same logical constant in $\mathbf{L}^{*}$ for distinct $\mathbf{L}$-systems.

\section{(2) A short sketch of logics}

In this section, I will sketch out logics to be dealt with in the present paper by their inference rules with respect to their logical constants. The logical constant NEGATION $\rightarrow$ is occasionally replaced by the prosositional constant CONTRADICTION $\wedge$. If we adopt $\wedge$ in place of $\rightarrow$, we have to replace the inference rules with respect to $\rightarrow$ by the inference rules with respect to $\wedge$. A number of logics are defined by inference rules with respect to their logical constants.

(2.1) Inference rules. The lower classical predicate logic LK is the strongest logic among popular logics such as LK itself, the intuitionistic pred- 
icate logic $\mathbf{L J}$, and the minimal predicate logic $\mathbf{L M}$ in the sense that it has all the inference rules with respect to all the usual logical constants $\rightarrow,($ ), $\wedge, \vee,(\exists), \neg$, and $\wedge$. The following is a list of inference rules described informally with respect to these logical constants.

I. One can deduce $\mathfrak{A} \rightarrow \mathfrak{B}$, if $\mathfrak{B}$ is deducible from the assumption $\mathfrak{A}$ or without the assumtpion $\mathfrak{R}$.

II. $\mathfrak{B}$ is deducible from $\mathfrak{A} \rightarrow \mathfrak{B}$ and $\mathfrak{A}$.

III. One can deduce $(x) \mathfrak{A}(x)$, if $\mathfrak{A}(h)$ is deducible for every $h$ i.e. for any new variable $h$ which does not occur in any assumption for $\mathfrak{Y}(h)$ nor in $(x) \mathfrak{H}(x)$. (By my notation: $\forall h !^{13)}$ )

IV. $\mathfrak{F}(h)$ is deducible from $(x) \mathfrak{l}(x)$.

v. One can deduce $\rightarrow \mathfrak{A}$, if $\mathfrak{B}$ and $\rightarrow \mathfrak{B}$ are deducible from the assumption $\mathfrak{A}$ or without the assumption $\mathscr{H}$.

VI. One can deduce $\mathfrak{A}$, if $\mathfrak{B}$ and $\rightarrow \mathfrak{B}$ are deducible from the assumption $\rightarrow \mathfrak{H}$ or without the assumption $\rightarrow \mathfrak{H}$.

VII. $\mathfrak{A} \wedge \mathfrak{B}$ is deducible from $\mathfrak{A}$ and $\mathfrak{B}$.

VIII. $\mathfrak{A}$ is deducible from $\mathfrak{H} \wedge \mathfrak{B}$ as well as from $\mathfrak{B} \wedge \mathfrak{?}$.

IX. $\mathfrak{A} \vee \mathfrak{B}$ is deducible from $\mathfrak{A}$ as well as from $\mathfrak{B}$.

X. $\mathfrak{A}$ is deducible from $\mathfrak{B} \vee \mathfrak{G}, \mathfrak{B} \rightarrow \mathfrak{A}$, and $(\mathfrak{s} \rightarrow \mathfrak{A}$.

XI. $(\exists x) \mathfrak{U}(x)$ is deducible from $\mathfrak{l}(h)$.

XII. One can take an $h$ satisfying $\mathfrak{H}(h)$ (by my notation: $\left.\exists h ! \mathfrak{A}(h)^{14)}\right)$, if $(\exists x) \mathfrak{A}(x)$ is deducible.

XIII. $\mathfrak{A}$ is deducible from $\wedge$.

XIV. $\mathfrak{A}$ is deducible from $(\mathfrak{Q} \rightarrow \mathfrak{B}) \rightarrow \mathfrak{A}$. (Peirce's rule ${ }^{15)}$ )

XV. $\mathfrak{H}$ is deducible from $(\mathfrak{A} \rightarrow \wedge) \rightarrow \wedge$. (reductio ad absurdum)

If we translate $\rightarrow \mathfrak{A}$ into $\mathfrak{A} \rightarrow \wedge$ or $\wedge$ into $\S \wedge \rightarrow \S$ for any fixed $\S$, Rules $\mathbf{V}$ and VI altogether become equivalent to Rules XIII and XIV altogether, as far

13) See ONO [1] and [3]. $\forall$ ! and $\exists !$ are called DENOMINATING QUANTIFIERS. It should be noticed that in reality we are interested in proving only such propositions that contain no free variables introduced by denominations.

14) See Foot-note 13).

15) See PEIRCE [1]. In my former work, ONO [1], I have adopted a more complicated form

XIV ${ }^{0}, \quad \mathfrak{A}$ is deducible from $\mathfrak{C} \rightarrow \mathfrak{A}$ and $(\mathfrak{E} \rightarrow \mathfrak{B}) \rightarrow \mathfrak{A}$

in place of XIV, because $\mathbf{X I V}^{0}$ seemed more convenient in practical usage. Anyway, $\mathbf{X I V}$ is equivalent to $\mathbf{X I V}^{0}$ as far as $\mathbf{I}$ and II are valịd. 
as Rules I and II are valid. However, if we compare them more in details, Rule XIII is equivalent to Rule $\mathbf{V}$ and

VIJ. One can deduce $\mathfrak{A}$ from $\mathfrak{B}$ and $\rightarrow \mathfrak{B}$. Accordingly, one should adopt Rule VIJ for LJ instead of Rule VI.

Rule XV can be regarded as superfluous because it is equivalent to the pair of Rules XIII and XIV as far as Rules I and II are valid. If I am mostly interested in the classical logic only, I might as well adopt this simple form in defining the logic straightforwardly. In the present paper, however, I am rather interested in development of various logics and in their mutual relations. The pair of Rules XIII and XIV look like more convenient for our present purpose.

(2.2 R) Peirce's rule that $(\mathfrak{A} \rightarrow \mathfrak{B}) \rightarrow \mathfrak{A}$ implies $\mathfrak{A}$ is just a modification of tertium non datur that $\mathfrak{H} \vee(\mathfrak{A} \rightarrow \wedge)$ holds, or in other form, that $(\mathfrak{A} \rightarrow \wedge) \rightarrow \mathfrak{A}$ implies $\mathfrak{A}$. I adopt Peirce's rule in defining $\mathbf{J}$-series logics not only because I would like to have the defining rule expressible in terms of $\rightarrow$ only, but essentially because I would like to have $\mathbf{L Q}$ as a sublogic of all the $\mathbf{K}$-series logics. (See (2.7 T).)

If I adopt $\mathfrak{A} \vee(\mathfrak{A} \rightarrow \hat{\wedge})$ or the rule that $(\mathfrak{U} \rightarrow \hat{\wedge}) \rightarrow \mathfrak{U}$ implies $\mathfrak{A}$ in place of Rule XIV, I would have Curry's LD instead of $\mathbf{L N}$. LD is a logic slightly weaker than $\mathbf{L N}$.

(2.3) The primitive logic LO is the logic having the inference rules I-IV only with respect to the two logical constants $\rightarrow$ and ( ). LO can be regarded as the simplest possible predicate logic. The lower classical predicate logic LK can be taken as the logic having the inference rules I-IV and VII-XIV with respect to the logical constants $\rightarrow,(), \wedge, \vee,(\exists)$, and $\wedge$. Other logics dealt with in this paper are logics lying between LO and LK.

(2.4) Table of logics dealt with in this paper characterized by means of their inference rules and their logical constants.

\begin{tabular}{|c|c|c|c|c|c|c|c|c|}
\hline \multirow{2}{*}{$\begin{array}{c}\text { Logical } \\
\text { systems }\end{array}$} & \multicolumn{3}{|c|}{ Logical constants } & \multicolumn{5}{c|}{ Inference rules } \\
\hline $\mathbf{L O}$ & $*$ & $\wedge, \vee,(\exists)$ & 人 & I-IV & VII-XII & XIII & XIV \\
\hline $\mathbf{L P}$ & $*$ & & $*$ & & $*$ & & & \\
$\mathbf{L Q}$ & $*$ & $*$ & & $*$ & $*$ & & \\
$\mathbf{L M}$ & $*$ & $*$ & $*$ & $*$ & $*$ & & $*$ \\
$\mathbf{L N}$ & $*$ & $*$ & $*$ & $*$ & $*$ & & \\
$\mathbf{L J}$ & $*$ & $*$ & $*$ & $*$ & $*$ & & $*$ \\
$\mathbf{L K}$ & $*$ & $*$ & $*$ & $*$ & $*$ & $*$ & $*$ \\
\hline
\end{tabular}


One can easily see that $\mathbf{L J}$ and $\mathbf{L K}$ defined here are equivalent to Gentzen's $\mathbf{L J}$ and $\mathbf{L} \mathbf{K}^{16)}$, respectively, if we identify $\rightarrow$ with $\rightarrow \wedge$.

(2.5 T) LO is a sublogic of LP.

Proof. LO is surely a logic weaker than LP, and LP is a logic weaker than $\mathbf{L J}$ which is a logic equivalent to Gentzen's LJ as has been pointed out in (2.4). Hence, any O-proposition $\mathfrak{A}$ provable in LP is provable in Gentzen's LJ. According to Gentzen's theorem ${ }^{17)}, \mathfrak{A}$ is provable by making use of $\mathbf{O}$ propositions only. Any proof of this kind can be followed in LO as can be checked without difficulty.

(2.6 T) LP is a sublogic of LM as well as of LJ. Namely, any P-proposition is provable in LP if and only if it is provable in LJ (or in LM).

Proof. Since LP is a logic weaker than $\mathbf{L M}$ and $\mathbf{L M}$ is a logic weaker than $\mathbf{L J}$, we have only to prove that any P-proposition $\mathfrak{A}$ provable in $\mathbf{L J}$ is provable in LP. Since LJ is equivalent to Gentzen's LJ as remarked in (2.4), $\mathfrak{A}$ is provable in Gentzen's $\mathbf{L} \mathbf{J}$ by making use of $\mathbf{P}$-propositions only. This proof of $\mathfrak{A}$ can be followed in LP as can be checked without difficulty.

(2.7 T) LQ is a sublogic of $\mathbf{L N}$ as well as of LK. Namely, any P.proposition is provable in $\mathbf{L Q}$ if and only if it is provable in $\mathbf{L K}$ (or in $\mathbf{L N}$ ).

Proof. Just as in the preceeding theorem, we have only to prove that any P-proposition $\mathfrak{A}$ provable in $\mathbf{L K}$ is provable in $\mathbf{L Q}$. Since $\mathbf{L K}$ is equivalent to Gentzen's $\mathbf{L K}$ as remarked in (2.4), $\mathfrak{A}$ is provable in Gentzen's LK by making use of P-propositions only. Hence, we have to check whether Rules I-IV, VIIXII, and XIV are powerful enough to show that all the Gentzen's inference rules $^{18)}$ except for those concerning NEGATION hold by suitable interpretation. I do not describe this check thoroughly here, I will exhibit here only an essential part of the examination; namely, the inference from $\Gamma, \mathfrak{A} \rightarrow \Delta, \mathfrak{B}$ to $\Gamma \rightarrow \Delta, \mathfrak{U} \supset \mathfrak{B}$ and the inference from $\Gamma \rightarrow \Delta, \mathfrak{A}(h)$ to $\Gamma \rightarrow \Delta,(x) \mathfrak{H}(x)$ assuming that $h$ does not occur in the latter sequent. These inferences in Gentzen's formulation can be interpreted in our system as the inference from $\mathfrak{\varsigma} \wedge \mathfrak{A} \rightarrow$

\footnotetext{
16) See GENTZEN [1].

17) See GENTZEN [1].

18) See GENTZEN [1].
} 
$\mathfrak{D} \vee \mathfrak{B}$ to $\mathfrak{S} \rightarrow \mathfrak{D} \vee(\mathfrak{A} \rightarrow \mathfrak{B})$ and the inference from $\mathfrak{S} \rightarrow \mathfrak{D} \vee \mathfrak{A}(h)$ to $\mathfrak{S} \rightarrow \mathfrak{D} \vee(x) \mathfrak{A}(x)$ assuming that $h$ does not occur in the latter proposition.

The first inference can be proved in $\mathbf{L Q}$ as follows: Assume $\wedge \mathfrak{A} \rightarrow \mathfrak{D} \vee \mathfrak{B}$. If we assume $\mathfrak{E}$, then $\mathfrak{A} \rightarrow \mathfrak{D} \vee \mathfrak{B}$ holds. Now, to prove $\mathfrak{D} \vee(\mathfrak{A} \rightarrow \mathfrak{B})$, it is enough to show $(\mathfrak{D} \vee(\mathfrak{A} \rightarrow \mathfrak{B}) \rightarrow \mathfrak{H}) \rightarrow \mathfrak{D} \vee(\mathfrak{A} \rightarrow \mathfrak{B})$ because Rule XIV holds in LQ. To show this, assume $\mathfrak{D} \vee(\mathfrak{A} \rightarrow \mathfrak{B}) \rightarrow \mathfrak{A}$. Then, $(\mathfrak{H} \rightarrow \mathfrak{B}) \rightarrow \mathfrak{A}$ holds, and this implies $\mathfrak{A}$ by Rule XIV. Hence, $\mathfrak{D} \vee \mathfrak{B}$ holds. On the other hand, $\mathscr{D} \rightarrow \mathfrak{D} \vee(\mathfrak{A} \rightarrow \mathfrak{B})$ as well as $\mathfrak{B} \rightarrow \mathfrak{D} \vee(\mathfrak{R} \rightarrow \mathfrak{B})$ is easily provable, so $\mathfrak{D} \vee(\mathfrak{P} \rightarrow \mathfrak{B})$ is provable too.

The second inference can be proved as follows: Assume that $(\leftarrow \rightarrow \mathfrak{D} \vee \mathfrak{A}(h)$ holds for any arbitrary variable $h$. To prove $\mathscr{D} \vee(x) \mathfrak{A}(x)$ in assuming $厄$, it is enough to show that $\mathfrak{D} \vee(x) \mathfrak{A}(x) \rightarrow(x) \mathfrak{A}(x)$ implies $\mathfrak{D} \vee(x) \mathfrak{A}(x)$, because Rule XIV holds in LQ. To show this, assume $\mathscr{D} \vee(x) \mathfrak{A}(x) \rightarrow(x) \mathfrak{A}(x)$. Then, $\mathfrak{D} \rightarrow(x) \mathfrak{A}(x)$ holds. Now, I show $(x) \mathfrak{A}(x)$. To show this, take any $g$. Then, $(5 \rightarrow \mathfrak{D} \vee \mathfrak{A}(g)$ holds by assumption, so $\mathscr{D} \vee \mathfrak{H}(g)$ holds. Now, $\mathfrak{D}$ implies $(x) \mathfrak{A}(x)$ which implies $\mathfrak{A}(g)$. So, $\mathfrak{A}(g)$ holds anyway. $(x) \mathfrak{A}(x)$ implies clearly $\mathfrak{D} \vee(x) \mathfrak{A}(x)$.

These are the only inference rules which need Rule XIV for proving them in $\mathbf{L Q .}$

(2.8 T) $\mathbf{~ L M ~ i s ~ i n t e r p r e t a b l y ~ e q u i v a l e n t ~ t o ~} \mathbf{L P}$ and $\mathbf{L N}$ is interpretably equivalent to $\mathbf{L Q}$.

Proof. According to (2.6 T), LP is a sublogic of LM, and the sublogic LP of $\mathbf{L M}$ can be faithfully interpreted in $\mathbf{L M}$ by virtue of (1.5 R). Accordingly, we have only to prove that $\mathbf{L M}$ can be faithfully interpreted in LP in proving the former half of the theorem. To show this, take any $\mathbf{L M}$-system $\mathbf{L M}(\alpha)$, and also take up a proposition symbol $C$ lying outside of $\alpha$. Let $\alpha$ be $\left\{A_{1}, \ldots, A_{k}\right\}, A_{i}$ being $\boldsymbol{n}(\boldsymbol{i})$-ary. Then, an embedding $\mathrm{E}$ of $\mathbf{L M}(\alpha)$ in $\mathbf{L P}(\alpha \cup\{C\})$ can be defined by

$$
\begin{aligned}
\mathrm{E}: \quad \rightarrow & \longrightarrow \rightarrow, \quad(\mathrm{r}) \longrightarrow(\mathrm{)}, \quad \wedge \rightarrow \wedge, \quad \vee \longrightarrow \vee, \\
& (\exists) \longrightarrow(\exists), \quad \wedge \longrightarrow C, \quad A_{i} \longrightarrow A_{i} .
\end{aligned}
$$

The latter half of the theorem can be also proved quite similarly, by making use of (2.7 T) in place of (2.6 T).

(2.9 T) LJ is interpretably equivalent to $\mathbf{L P}$, and $\mathbf{L K}$ is interpretably equivalent to $\mathbf{L Q}$. 
Proof. According to (2.6 T), LP is a sublogic of $\mathbf{L J}$, and the sublogic $\mathbf{L P}$ of $\mathbf{L J}$ can be faithfully interpreted in $\mathbf{L J}$ by virtue of (1.5 R). Accordingly, we have only to prove that $\mathbf{L J}$ can be faithfully interpreted in LP in proving the former half of the theorem. To show this, take any $\mathbf{L J}$-system $\mathbf{L J}(\alpha)$, and also take up a propositional symbol $C$ lying outside of $\alpha$. Let $\alpha$ be $\left\{A_{1}, \ldots, A_{k}\right\}$, $A_{i}$ being $n(i)$-ary. Then, an embedding $\mathbf{E}$ of $\mathbf{L J}(\alpha)$ in $\mathbf{L P}(\alpha \cup\{C\})$ can be defined by

$$
\begin{aligned}
\mathrm{E}: \quad & \rightarrow \rightarrow, \quad() \longrightarrow(), \quad \wedge \longrightarrow \wedge, \quad \vee \longrightarrow \vee, \\
& (\exists) \longrightarrow(\exists), \quad \wedge \longrightarrow \mathfrak{F}, \quad A_{i} \longrightarrow A_{i},
\end{aligned}
$$

where $\mathfrak{F}$ stands for the proposition

$$
C \wedge\left(\sigma_{1}\right) A_{1}\left(\sigma_{1}\right) \wedge \cdots \wedge\left(\sigma_{k}\right) A_{k}\left(\sigma_{k}\right),
$$

each $\sigma_{i}$ being a sequence of mutually distinct bound variables of length $n(i)$.

The latter half of the theorem can be proved quite similarly, by making use of $(2.7 \mathbf{T})$ in place of $(2.6 \mathbf{T})$.

$(2.10 \mathrm{R})$ By virtue of $(2.8 \mathrm{~T})$ and $(2.9 \mathrm{~T})$, we can see that all the $\mathbf{J}$ series logics are interpretably equivalent to each other and also that all the $\mathbf{K}$-series logics are interpretably equivalent to each other.

\section{(3) Elementary properties of $\mathfrak{R}$-closures and $\mathfrak{\Re}$-closed propositions}

In this section, we study the $\mathfrak{R}$-CLOSURE $\mathfrak{A}^{\Re}$ of any proposition $\mathfrak{A}$ and $\mathfrak{R}$ CLOSED PROPOSITIONS for any $n$-ary relation $\mathfrak{R}(\boldsymbol{n}=0,1,2, \ldots)$. The logic $\mathbf{L}$ we stand on in this section can be taken as LO. More precisely, $\mathbf{L}$ may have logical constants other than $\rightarrow$ and ( ), but the inference rules I-IV only are assumed in this section.

All the theorems in this section except for $(3.12 \mathbf{T})$ hold for any $n$-ary relation $\Re$ in general. Theorem (3.12 T) discloses a distinctive feature of the case $n=0$. In the following, I will denote by $\xi$ a sequence of mutually distinct bound variables of length $n$ and by $\eta$ as well as by $\zeta$ a sequence of mutually distinct free variables of length $n$.

(3.1 D) $\mathfrak{M}^{\Re} \equiv(\xi)((\mathfrak{U} \rightarrow \Re(\xi)) \rightarrow \Re(\xi))$.

$\mathfrak{U}^{\Re}$ is called the $\mathfrak{R}$-CLOSURE of $\mathfrak{A}$.

(3.2 D) $\mathfrak{A}$ is called $\mathfrak{R}$-CLOSED if and only if $\mathfrak{A}^{\mathfrak{R}}$ implies $\mathfrak{A}$, 
(3.3 T) $\mathfrak{U}$ implies $\mathfrak{U}^{\Re}$.

(3.4 T) $\mathfrak{A} \rightarrow \mathfrak{B}$ implies $\mathfrak{I}^{\Re} \rightarrow \mathfrak{B}^{\Re}$.

Proof. Assume $\mathfrak{A} \rightarrow \mathfrak{B}$. Then, I will prove $\mathfrak{B}^{\mathfrak{R}}$ by assuming $\mathfrak{U}^{\mathfrak{R}}$ i.e. $(\xi)((\mathfrak{A} \rightarrow \mathfrak{R}(\xi)) \rightarrow \mathfrak{N}(\xi))$. To prove $\mathfrak{B}^{\Re}$ take any $\zeta$ satisfying $\mathfrak{B} \rightarrow \mathfrak{N}(\zeta)$. Since we assume $\mathfrak{A} \rightarrow \mathfrak{B}$, holds $\mathfrak{A} \rightarrow \mathfrak{N}(\zeta)$. On the other hand, $(\mathfrak{A} \rightarrow \mathfrak{N}(\zeta)) \rightarrow \mathfrak{R}(\zeta)$ holds by assumption, so we have $\mathfrak{R} \zeta$ ). Hence, $\mathfrak{I}^{\Re} \rightarrow \mathfrak{B}^{\Re}$ holds.

(3.5 T) $\mathfrak{I}^{\Re}$ is $\mathfrak{R}$-closed. $\mathfrak{R}(\zeta)$ is $\mathfrak{R}$-closed.

Proof. I will prove at first that $\mathfrak{U}^{\Re \Re}$ implies $\mathfrak{U}^{\Re}$. Namely, assume $\mathfrak{U}^{\Re \Re}$. To prove $\mathfrak{H}^{\Re}$, take any $\zeta$ satisfying $\mathfrak{H} \rightarrow \mathfrak{R}(\zeta)$. Then, $\mathfrak{U}^{\Re} \rightarrow \Re(\zeta)$ holds, since $\mathfrak{A}^{\mathfrak{R}}$ implies $(\mathfrak{A} \rightarrow \mathfrak{R}(\zeta)) \rightarrow \mathfrak{R}(\zeta)$. On the other hand, the assumption $\mathfrak{A}^{\mathfrak{R} \Re}$ implies $\left(\mathfrak{A}^{\Re} \rightarrow \mathfrak{A}(\zeta)\right) \rightarrow \mathfrak{R}(\zeta)$. So, we have $\mathfrak{R}(\zeta)$.

To prove next that $\Re(\zeta)$ is $\Re$-closed, assume $\mathfrak{R}^{\Re}(\zeta)$ i.e. $(\xi)((\Re(\zeta) \rightarrow \mathfrak{R}(\xi)) \rightarrow$ $\Re(\xi))$. Then, we have easily $\Re(\zeta)$.

(3.6 R) By virtue of $(3.3 \mathbf{T})$ and $(3.5 \mathbf{T})$, any proposition $\mathfrak{A}$ is $\mathfrak{R}$-closed if and only if it is expressible in the form $\mathfrak{U}^{\mathfrak{R}}$.

(3.7 T) $\quad(\mathfrak{A} \rightarrow \mathfrak{B})^{\mathfrak{R}}$ implies $\mathfrak{A}^{\mathfrak{R}} \rightarrow \mathfrak{B}^{\mathfrak{R}}$.

Proof. I will prove $\mathfrak{B}^{\Re}$ by assuming $(\mathfrak{H} \rightarrow \mathfrak{B})^{\Re}$ and $\mathfrak{A}^{\Re}$. To prove $\mathfrak{B}^{\Re}$, take any $\zeta$ satisfying $\mathfrak{B} \rightarrow \mathfrak{R}(\zeta)$. Then, $\mathfrak{A} \rightarrow \mathfrak{B}$ implies $\mathfrak{A} \rightarrow \mathfrak{A}(\zeta)$. On the other hand, $\mathfrak{A} \rightarrow \mathfrak{N}(\zeta)$ implies $\mathfrak{R}(\zeta)$ because $\mathfrak{A}^{\mathfrak{R}}$ is assumed. So, $\mathfrak{A} \rightarrow \mathfrak{B}$ implies $\mathfrak{N}(\zeta)$. Now, $(\mathfrak{A} \rightarrow \mathfrak{B}) \rightarrow \mathfrak{R}(\zeta)$ implies $\mathfrak{R}(\zeta)$ because $(\mathfrak{A} \rightarrow \mathfrak{B})^{\mathfrak{R}}$ is assumed. So, $\mathfrak{R}(\zeta)$ holds. $\mathfrak{B}^{\Re}$ is thus proved.

$(3.8 \mathrm{~T}) \quad(x)^{\mathfrak{R}} \mathfrak{A}(x)$ implies $(x) \mathfrak{Q}^{\Re}(x)^{19)}$.

Proof. Assume $(x)^{\mathfrak{R}} \mathfrak{A}(x)$. To prove $(x) \mathfrak{A}^{\mathfrak{R}}(x)$ i.e. $(x)(\zeta)((\mathfrak{A}(x) \rightarrow \mathfrak{R}(\zeta)) \rightarrow$ $\Re(\zeta)$, take any $h$ and $\zeta$ satisfying $\mathfrak{A}(h) \rightarrow \mathfrak{N}(\zeta)$. Then, $(x) \mathfrak{A}(x) \rightarrow \mathfrak{N}(\zeta)$ holds.

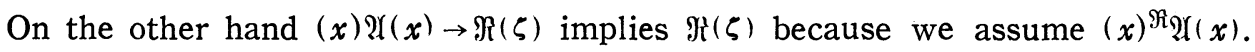
Hence $\mathfrak{R}(\zeta)$ holds. $(\boldsymbol{x}) \mathfrak{A}^{\mathfrak{R}}(\boldsymbol{x})$ is thus proved.

(3.9 R) I can not expect commutativity of the $\Re$-closure operation with the logical operations $\rightarrow$ and ( ). However, we can see by virtue of $(3.3 \mathbf{T})-$ (3.8 T) that the class of $\mathfrak{R}$-closed propositions is closed for the $\Re$-closure

19) Formulas of the forms $\mathscr{N} \Re(p)$ and $(x) \Re \mathfrak{A}(x)$ stand naturally for $(\mathfrak{A}(p))^{\Re}$ and $((x) \mathscr{Y}(x))^{\mathfrak{R}}$, respectively. 
operation and the $\Re$-closure operation commutes with the logical constants $\rightarrow$ and $($ ) in the class of $\mathfrak{R}$-closed propositions.

(3.10 $\mathbf{T}) \quad(\xi)(\mathfrak{A} \rightarrow \mathfrak{N}(\xi))$ is $\mathfrak{R}$-closed.

Proof. Assume $(\xi)^{\Re}(\mathfrak{A} \rightarrow \Re(\xi))$. Then, by virute of $(3.8 \mathrm{~T}),(\xi)(\mathfrak{A} \rightarrow \mathfrak{R}(\xi))^{\Re}$ holds. Now, we prove $(\xi)(\mathfrak{A} \rightarrow \mathfrak{R}(\xi))$. To prove this, take any $\zeta$. Then, $(\mathfrak{A} \rightarrow \mathfrak{R}(\zeta))^{\mathfrak{R}}$ i.e. $(\eta)(((\mathfrak{A} \rightarrow \mathfrak{R}(\zeta)) \rightarrow \mathfrak{R}(\eta)) \rightarrow \mathfrak{R}(\eta))$ holds. Hence, $\left.(\mathfrak{A} \rightarrow \mathfrak{R}(\zeta)) \rightarrow \mathfrak{R}(\zeta)\right)$ implies $\mathfrak{A}(\zeta)$. $(\mathfrak{A} \rightarrow \mathfrak{R}(\zeta)) \rightarrow \mathfrak{R}(\zeta)$ holds if we assume $\mathfrak{A}$. Hence, $\mathfrak{A} \rightarrow \mathfrak{R}(\zeta)$ holds for any $\zeta$.

(3.11 T) ( $(\xi) \Re(\xi)$ implies every $\mathfrak{R}$-closed proposition.

(3.12 T) Let $\mathfrak{F}$ be any proposition. Then, $(\mathfrak{A} \mathfrak{F} \rightarrow \mathfrak{B} \mathfrak{F}) \rightarrow \mathfrak{H} \mathfrak{F}$ implies $\mathfrak{H} \mathfrak{F}$, regarding $\mathfrak{F}$ as a 0 -ary relation. Namely Peirce's rule holds for $\mathfrak{F}$-closed propositions.

Proof. Assume $(\mathfrak{U} \mathfrak{F} \rightarrow \mathfrak{B} \mathfrak{F}) \rightarrow \mathfrak{U} \mathfrak{F}$. To show $\mathfrak{Y} \mathfrak{F}$, assume $\mathfrak{A} \rightarrow \mathfrak{F}$. Then, $\mathfrak{V}^{\mathfrak{F}}$ implies $\widetilde{f}$ evidently. On the other hand, according to $(3.5 \mathbf{T})$ and $(3.11 \mathrm{~T})$ (taking $n=0), \mathfrak{F}$ implies $\mathfrak{B} \mathfrak{F}$. Hence, $\mathfrak{A} \mathfrak{F} \rightarrow \mathfrak{B} \mathfrak{F}$ holds. Since $(\mathfrak{A} \mathfrak{F} \rightarrow \mathfrak{B} \mathfrak{F}) \rightarrow \mathfrak{A} \mathfrak{F}$ is assumed, we have $\mathfrak{2} \mathfrak{F}$. So, we have $\mathfrak{F}$.

Peirce's rule holds for $\mathfrak{F}$-closed propositions by virtue of the above mentioned fact and (3.6 R).

\section{(4) Logical constants relative to relations}

Outline of this section: With respect to any $n$-ary relation $\Re$, I will define the logical constants $\Re$-CONJUNCTION $\underset{\Re}{\wedge}$, $\Re$-DISJUNCTION $\underset{\Re}{\vee}$, and $\Re$-EXISTENTIAL QUANTIFICATION $(\underset{\Re}{\exists})$. With respect to $\underset{\Re}{\wedge}, \underset{\Re}{\vee}$, and $(\underset{\Re}{\exists})$ together with the logical constants $\rightarrow$ and ( ), the class of $\Re$-closed propositions is closed. Inference rules I-IV and VII-XII hold for $\Re$-closed propositions with respect to $\rightarrow,($ ), $\underset{\Re}{\wedge}, \underset{\Re}{\vee}$, and $(\underset{\Re}{\exists})$. If $\mathfrak{R}$ is an $\mathbf{O}$-relation, $\mathfrak{A} \underset{\Re}{\wedge} \mathfrak{B}$ as well as $\mathfrak{A} \vee \mathfrak{B}$ is an $\mathbf{O}$-proposition for any $\mathbf{O}$-propositions $\mathfrak{A}$ and $\mathfrak{B}$, and $(\underset{\mathfrak{R}}{\exists} x) \mathfrak{A}(x)$ is an $\mathbf{O}$-proposition for any $\mathbf{O}$-proposition $\mathfrak{U}(p)$. The proof that inference rules I-IV and VII-XII hold for $\Re$-closed propositions with respect to $\rightarrow,(), \wedge_{\Re}$, $\underset{\Re}{\vee}$, and $(\underset{\Re}{\exists})$ can be carried out in LO.

In this section, I assume only the inference rules I-IV, unless specially noticed otherwise. 
(4.1 D) Let $\mathfrak{R}$ be any $n$-ary relation, and $\xi$ be a sequence of $n$ mutually distinct variables which occur neither in $\mathfrak{A}$ nor in $\mathfrak{B}$ nor in $\mathfrak{A}(x)$. Then, I define $\underset{\Re}{\wedge}, \underset{\Re}{\bigvee}$, and $\underset{\Re}{\exists}$ ) by

$$
\begin{aligned}
& \mathfrak{A} \wedge \mathfrak{R} \equiv(\xi)((\mathfrak{A} \rightarrow(\mathfrak{B} \rightarrow \mathfrak{R}(\xi))) \rightarrow \mathfrak{R}(\xi)),
\end{aligned}
$$

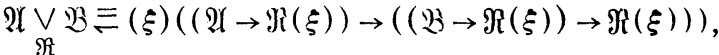

$$
\begin{aligned}
& (\underset{\mathfrak{K}}{\exists} x) \mathfrak{U}(x) \equiv(\xi)((x)(\mathfrak{\mathscr { U }}(x) \rightarrow \mathfrak{R}(\xi)) \rightarrow \mathfrak{R}(\xi)) .
\end{aligned}
$$

The logical constants $\wedge_{\Re}, \underset{\Re}{\vee}$, and $(\underset{\Re}{\exists})$ are called $\Re$-CONJUNCTION, $\Re$-DISJUNCTION, and $\Re$-EXISTENTIAL QUANTIFICATION, respectively.

(4.2 R) Let $\mathbf{R}$ be any $O$-relation, According to the definitions of $\wedge_{\Re}$ and $\vee \mathfrak{\Re}, \mathfrak{A} \wedge \mathfrak{R}$ as well as $\mathfrak{A} \vee \mathfrak{R}$ is an $\mathbf{O}$-proposition for any $\mathbf{O}$-propositions $\mathfrak{A}$ and $\mathfrak{B}$. According to the definition of $(\underset{\Re}{\exists}),(\underset{\Re}{\exists} x) \mathfrak{U}(x)$ is an $\mathbf{O}$-proposition for any o-proposition $\mathfrak{A}(p)$.

(4.3 T) $\mathfrak{A} \underset{\Re}{\wedge} \mathfrak{B}, \mathfrak{H} \underset{\Re}{\vee} \mathfrak{B}$, and $\underset{\mathfrak{R}}{\exists} x) \mathfrak{A}(x)$ are all $\mathfrak{R}$-closed for any relation $\mathfrak{R}$.

Proof. For $\underset{\Re}{\wedge}$ as well as $(\underset{\Re}{\exists})$, we can prove this theorem easily by making use of $(3.10 \mathrm{~T})$ and $(4.1 \mathrm{D})$. For $\underset{\Re}{\vee}$, we had better prove the generalized theorem of $(3.10 \mathrm{~T})$ that

$$
(\xi)(\mathfrak{A} \rightarrow(\mathfrak{B} \rightarrow(\quad \cdot \quad \cdot \quad \cdot(\widetilde{B} \rightarrow \mathfrak{R}(\xi)) \cdots))
$$

is $\mathfrak{R}$-closed, beforehand. Then, we can prove the theorem just as we proved (3.10 T).

(4.4 T) $\mathfrak{A}$ and $\mathfrak{B}$ imply $\mathfrak{\mathfrak { U }} \underset{\mathfrak{R}}{\wedge} \mathfrak{B}$.

Proof. Assume $\mathfrak{A}$ and $\mathfrak{B}$. To prove $\mathfrak{A} \wedge \mathfrak{B}$ i.e. $(\xi)((\mathfrak{A} \rightarrow(\mathfrak{B} \rightarrow \mathfrak{H}(\xi))) \rightarrow \mathfrak{R}(\xi))$, take any $\zeta$ satisfying $\mathfrak{U} \rightarrow(\mathfrak{B} \rightarrow \mathfrak{R}(\zeta))$. Then, $\mathfrak{R}(\zeta)$ holds since we assume $\mathfrak{A}$ and $\mathfrak{B}$. Hence, $\mathfrak{A} \wedge \mathfrak{\Re} \mathfrak{B}$ holds.

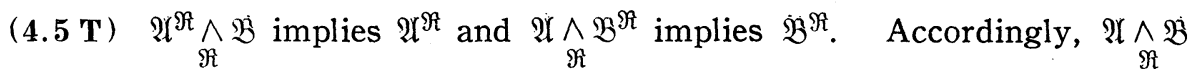
implies $\mathfrak{A}$ as well as $\mathfrak{B}$ for any $\mathfrak{R}$-closed $\mathfrak{A}$ and $\mathfrak{B}$.

Proof. I will prove only that $\mathfrak{I}_{\mathfrak{R}}^{\mathfrak{A}} \wedge \mathfrak{B}$ implies $\mathfrak{U}^{\mathfrak{R}}$. To show this, assume $\mathfrak{A}^{\Re} \wedge \mathfrak{B}$ and take any $\zeta$ satisfying $\mathfrak{A} \rightarrow \mathfrak{R}(\zeta)$. Then, $\mathfrak{H}^{\Re}$ implies $\mathfrak{N}(\zeta)$, because

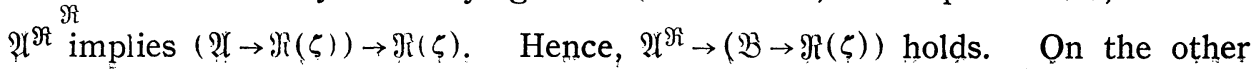


hand, $\left(\mathfrak{H}^{\mathfrak{R}} \rightarrow(\mathfrak{B} \rightarrow \mathfrak{R}(\zeta))\right) \rightarrow \mathfrak{R}(\zeta)$ holds by assumption, so $\mathfrak{R}(\zeta)$ holds. Thus, $\mathfrak{U}^{\mathfrak{R}}$ is proved.

(4.6 T) $\mathfrak{A}$ as well as $\mathfrak{B}$ implies $\mathfrak{U} \underset{\mathfrak{R}}{\vee} \mathfrak{B}$.

Proof. I will prove only that $\mathfrak{A}$ implies $\mathfrak{A} \underset{\mathfrak{R}}{\vee} \mathfrak{B}$ i.e. $(\xi)((\mathfrak{A} \rightarrow \mathfrak{R}(\xi)) \rightarrow((\mathfrak{B} \rightarrow$ $\Re(\xi)) \rightarrow \mathfrak{R}(\xi))$ ). To prove this, assume $\mathfrak{U}$ and take any $\zeta$ satisfying $\mathfrak{U} \rightarrow \mathfrak{R}(\zeta)$. Then, $\mathfrak{R}(\zeta)$ holds as far as $\mathfrak{A}$ holds. Hence, $(\mathfrak{B} \rightarrow \mathfrak{R}(\zeta)) \rightarrow \mathfrak{R}(\zeta)$ holds as far as $\mathfrak{U}$ holds. Therefore $\mathfrak{U} \vee \mathfrak{B}$ holds.

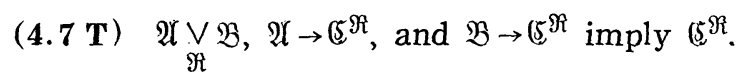

Proof. Assume $\mathfrak{A} \underset{\mathfrak{R}}{\vee \mathfrak{B}}, \mathfrak{U} \rightarrow \mathfrak{S}^{\mathfrak{R}}$, and $\mathfrak{B} \rightarrow \mathfrak{S}^{\mathfrak{R}}$. Then, I will prove at first $\widetilde{(}^{\Re \Re}$. To prove this, take any $\zeta$ satisfying $\zeta^{\Re} \rightarrow \mathfrak{N}(\zeta)$. Then, $\mathfrak{A} \rightarrow \mathfrak{R}(\zeta)$ as well as $\mathfrak{B} \rightarrow \mathfrak{R}(\zeta)$ holds because we assume $\mathfrak{A} \rightarrow \mathfrak{S}^{\Re}$ and $\mathfrak{B} \rightarrow \mathfrak{C}^{\Re}$. On the other hand, $\mathfrak{U} \rightarrow \mathfrak{R}(\zeta)$ and $\mathfrak{B} \rightarrow \mathfrak{R}(\zeta)$ imply $\mathfrak{R}(\zeta)$ because we assume $\mathfrak{X} \vee \mathfrak{R}$ i.e. $(\xi)((\mathfrak{A} \rightarrow \mathfrak{R}(\xi)) \rightarrow$ $((\mathfrak{B} \rightarrow \Re(\xi)) \rightarrow \mathfrak{R}(\xi)))$. $\left(\Im^{\Re \Re}\right.$ implies $\mathfrak{S}^{\Re}$ by virtue of $(3.5 \mathbf{T})$.

(4.8 T) $\mathfrak{A}(h)$ implies $\underset{\mathfrak{A}}{(\exists x}) \mathfrak{U}(x)$.

Proof. Assume $\mathfrak{A}(h)$. To show $\underset{\mathfrak{R}}{\exists x} \mathfrak{\exists} \mathfrak{A}(x)$, take any $\zeta$ satisfying $(x)(\mathfrak{A}(x) \rightarrow$ $\mathfrak{N}(\zeta)$ ). Then, $\Re(\zeta)$ holds, because $\mathfrak{H}(h) \rightarrow \Re(\zeta)$ holds by assumption.

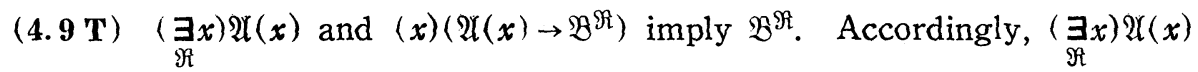
implies any $\mathfrak{R}$-closed $\mathfrak{B}$ satisfying $(\boldsymbol{x})(\mathfrak{A}(\boldsymbol{x}) \rightarrow \mathfrak{B})$.

Proof. Assume $(\underset{\Re}{\exists} x) \mathfrak{U}(x)$ and $(x)\left(\mathfrak{U}(x) \rightarrow \mathfrak{B}^{\Re}\right)$. Then, I prove at first $\mathfrak{B}^{\Re \Re}$. To prove this, take any $\zeta$ satisfying $\mathfrak{B}^{\Re} \rightarrow \mathfrak{R}(\zeta)$. I will prove now $(x)(\mathfrak{A}(x) \rightarrow$ $\mathfrak{R}(\zeta)$ ). To prove this, take any $h$ satisfying $\mathscr{U}(h)$. Then, $\mathfrak{A}(h) \rightarrow \mathfrak{B}^{\Re}$ holds because we assume $(x)\left(\mathfrak{H}(x) \rightarrow \mathfrak{B}^{\Re}\right)$. Accordingly, $\mathfrak{H}(h)$ implies $\mathfrak{R}(\zeta)$. So, $(x)(\mathfrak{A}(x) \rightarrow \mathfrak{R}(\zeta))$ holds. On the other hand, the assumption $(\underset{\Re}{\exists}) \mathfrak{R}(x)$ i.e. $(\xi)((x)(\mathfrak{H}(x) \rightarrow \mathfrak{N}(\xi)) \rightarrow \mathfrak{R}(\xi))$ implies $(x)(\mathfrak{H}(x) \rightarrow \mathfrak{R}(\zeta)) \rightarrow \mathfrak{R}(\zeta)$. Hence, $\mathfrak{R}(\zeta)$ holds. $\mathfrak{B}^{\Re \Re}$ is thus proved. According to $(3.5 \mathbf{T}), \mathfrak{B}^{\Re \Re}$ implies $\mathfrak{B}^{\Re}$.

(4.10 R) With respect to the logical constants $\underset{\Re}{\wedge}, \underset{\Re}{\vee}$, and $(\underset{\Re}{\exists})$ together with the original logical constants $\rightarrow$ and ( ), Rules I-IV and VII-XII can be employed for $\Re$-closed propositions for any relation $\Re$. Furthermore, Rule XIV can be employed for $\Re$-closed propositions for any O-ARY RELATION $\Re$.

Illustration for Rules $\mathbf{I}-\mathbf{I V}$ and VII-XI would be needless for any relation 
$\Re$. As for Rule XII, the DENOMINATING QUANTIFICATION $\underset{\Re}{\exists}$ ! must be suitably introduced ${ }^{30)}$. For any $\mathbf{O}$-relation $\Re, I$ assert that any proof employing Rules VII-XII for $\underset{\Re}{\wedge}, \underset{\Re}{\vee}$, and $(\underset{\Re}{\exists})$ together with Rules I-IV for $\rightarrow$ and ( ) can be transformed into a right proof in LO. (4.4 T)-(4.8 T) indicate that this is the case for Rules VII-XI. When Rule XII is employed for $\underset{\Re}{\exists} x) \mathfrak{A}(x)$, we denominate a free variable, say $h$, satisfying $\mathfrak{U}(h)$ under the assumption $(\exists x) \mathfrak{A}(x)$. As far as the denomination is valid, we can assert $(x)(\mathfrak{U}(x) \rightarrow \mathfrak{B}(x))$ instead of the original assertion $\mathfrak{B}(h)$. If the original assertion $\mathfrak{B}(h)$ becomes independent of $h$, then we can assert $\mathfrak{B}(h)$, becasue $(x)(\mathfrak{A}(x) \rightarrow \mathfrak{B}(x))$ and $(\underset{\Re}{\exists} x) \mathfrak{U}(x)$ imply $\mathfrak{B}(h)$ by $(4.9 \mathbf{T})$ for any $\mathfrak{R}$-closed $\mathfrak{B}(h)$.

By virtue of $(3.12 \mathbf{T})$, Rule XIV holds for $\mathfrak{F}$-closed propositions for any proposition $\mathfrak{F}$ regarding it as a 0 -ary relation.

$(4.11 \mathrm{R})$ Let us now consider the matter in $\mathbf{J}$ - and $\mathbf{K}$-series logics. Even in these logics, the $\Re$-closure operation and the new logical constants $\underset{\Re}{\wedge}, \underset{\Re}{V}$, and $(\underset{\Re}{\exists})$ can be introduced in the same way as in $(4.1 \mathrm{D})$ for any relation $\Re$. In $\mathbf{L P}$ as well as in $\mathbf{L Q}$, we can show without difficulty that

$$
\begin{aligned}
& \mathfrak{U}^{\Re} \wedge \mathfrak{B}^{\Re} \equiv \mathfrak{U}^{\Re} \wedge \mathfrak{B}^{\Re}, \\
& \mathfrak{U}^{\Re} \stackrel{\Re}{\vee} \mathfrak{B}^{\Re} \equiv \mathfrak{U}^{\Re} \vee \mathfrak{B}^{\Re}, \\
& (\underset{\mathfrak{R}}{\exists} x) \mathfrak{U}^{\Re}(x) \equiv(\exists x) \mathfrak{U}^{\Re}(x)^{21)}
\end{aligned}
$$

are all provable as $(3.9 \mathrm{R})$ and $(4.10 \mathrm{R})$ suggest it.

(5) Interpretable equivalence of J-series logics to LO and faithful interpretations of $\mathrm{K}$-series logics in $\mathrm{LO}$

Any $\mathbf{J}$-series logic can be proved interpretably equivalent to the primitive logic LO, and any $\mathbf{K}$-series logic can be proved faithfully interpretable in the same logic LO. According to $(2.10 \mathbf{R})$, any $\mathbf{J}$-series logic is interpretably equivalent to $\mathbf{L P}$ and $\mathbf{K}$-series logic is interpretably equivalent to $\mathbf{L Q}$, so we have only to prove that $\mathbf{L P}$ is interpretably equivalent to $\mathbf{L O}$ and that $\mathbf{L Q}$ can

20) As for details of the denominating quantifications, see ONO [1] and [3]. Here we deal with proofs naturally containing variables introduced by denominations, but only proofs of propositions containing no variables introduced by denominations. See Footnotes 13)-14).

21) Any proposition of the form $\mathfrak{A} \equiv \mathfrak{B}$ denotes naturally $(\mathfrak{A} \rightarrow \mathfrak{B}) \wedge(\mathfrak{B} \rightarrow \mathfrak{A})$ as far as $\wedge$ is defined in the logic. 
be faithfully interpreted in LO.

(5.1 T) LP is interpretably equivalent to LO.

Proof. According to (2.5 T), LO is a sublogic of LP. Hence, according to $(1.5 \mathrm{R})$, the sublogic LO of LP can be faithfully interpreted in LP. Consequently, we have only to prove that LP can be faithfully interpreted in LO.

To show this, let us take up any $\mathbf{L P}$-system $\mathbf{L P}(\alpha)$. Let $\alpha$ be $\left\{A_{1}, \ldots, A_{k}\right\}$, every $A_{i}$ be $n(i)$-ary relation, and $n$ be the maximum number of $n(i)$ 's. Let us further take up any $(n+1)$-ary relation symbol $R$, which lies naturally outside of $\alpha$.

Now, I can define an embedding $\mathbf{E}$ of $\mathbf{L P}(\boldsymbol{\alpha})$ into $\mathbf{L O}(\alpha \cup\{R\})$ by the following rule of associations:

$$
\mathrm{E}: \quad \rightarrow \longrightarrow \rightarrow, \quad\left(\mathrm { ) } \longrightarrow \left(\mathrm{)}, \wedge \longrightarrow \wedge_{R}, \quad \vee \longrightarrow \underset{R}{\vee}, \quad(\exists) \longrightarrow(\underset{R}{\exists}), \quad A_{i} \longrightarrow A_{i}^{R}\right.\right.
$$

Naturally, $A_{i}^{R}$ denotes an $\boldsymbol{n}(i)$-ary relation defined by

$$
A_{i}^{R}\left(p_{1}, \ldots, p_{n i i}\right) \equiv\left(A_{i}\left(p_{1}, \ldots, p_{n(i)}\right)\right)^{R} .
$$

Now, let $\mathfrak{U}$ be any proposition (naturally a P-proposition) of $\mathbf{L P}(\alpha)$, and $\mathfrak{U}[R]$ be its word-for-word translation in $\operatorname{LO}(\alpha \cup\{R\})$ by $E$. By making use of (3.9R), $(4.3 \mathrm{~T})$, and $(4.10 \mathrm{R})$, we can easily prove that $\mathfrak{U}[R]$ is provable in $\mathbf{L O}(\alpha \cup\{R\})$ as far as $\mathfrak{A}$ is provable in $\mathbf{L P}(\alpha)$.

Next, I prove that $\mathfrak{U}$ is provable in $\mathbf{L P}(\alpha)$ as far as $\mathfrak{X}[R]$ is provable in $\mathbf{L O}(\alpha \cup\{R\})$. To prove this, let us assume that $\mathfrak{x}[R]$ is provable in $\operatorname{LO}(\alpha \cup\{R\})$ by a proof $\Pi$. Since LO is a logic weaker than LP, $I$ is a right proof of $\mathfrak{R}[R]$ in $\mathbf{L P}(\boldsymbol{\alpha} \cup\{R\})$. Now, take up $k+1$ mutually distinct free variables $a_{1}, \ldots, a_{k}$, and $d$ which do not occur in $\Pi$. We can now define a word-forword translation $T$ of the whole proof $I$ and the proposition $\mathfrak{U}$ by

$$
\begin{aligned}
\mathrm{T}: \rightarrow & \longrightarrow \rightarrow,() \rightarrow(), \wedge \longrightarrow \wedge, \vee \rightarrow \vee, \quad(\exists) \longrightarrow(\exists), \\
& A_{i}\left(p_{1}, \ldots, p_{n(i)}\right) \rightarrow R(a_{i}, p_{1}, \ldots, p_{n(i)}, \underbrace{d, \ldots, d)}_{n-4(i)} \\
& R\left(p_{0}, p_{1}, \ldots, p_{n}\right) \longrightarrow R\left(p_{0}, p_{1} \ldots, p_{n}\right) .
\end{aligned}
$$

Let $\mathfrak{U}^{*}, \mathfrak{Y}^{*}[R]$, and $\Pi^{*}$ be the translations of $\mathfrak{A}, \mathfrak{U}[R]$, and $\Pi$ by $\mathrm{T}$, respectively. Then, we can easily see that $\Pi^{*}$ is a right proof of $\mathfrak{A}^{*}[R]$ in $\operatorname{LP}(\{R\})$. We can also see easily that $\mathfrak{U}^{*}[R]$ is the word-for-word translation of $\mathfrak{A}^{*}$ by the translation $\mathrm{S}$ defined by

$$
\mathrm{S}: \quad \rightarrow \longrightarrow \rightarrow, \quad\left(\mathrm{)} \longrightarrow(\mathrm{)}), \wedge \longrightarrow \wedge_{R}, \quad \vee \longrightarrow \underset{R}{\vee}, \quad(\exists) \longrightarrow(\underset{R}{(\exists}), \quad R \longrightarrow R^{R}\right.
$$


Then, by virtue of $(3.6 \mathbf{R}),(3.9 \mathrm{R})$, and $(4.11 \mathbf{R}), \mathfrak{U}^{*}$ must be equivalent to $\mathfrak{R}^{*}[R]$ in $\operatorname{LP}(\{R\})$. Hence, $\mathfrak{A}^{*}$ must be provable in $\operatorname{LP}(\{R\})$. Now, taking into consideration the situation that $\mathfrak{I}^{*}$ consists of elementary propositions exclusively of the form $R\left(a_{i}, p_{1}, \ldots, p_{n(i)}, d, \ldots, d\right)$, we can prove in LP also the proposition obtained on replacing every elementary proposition $R\left(a_{i}, p_{1}, \ldots\right.$, $\left.p_{n(i)}, d, \ldots, d\right)$ by $A_{i}\left(p_{1}, \ldots, p_{n(i)}\right)$, which is nothing but the proposition $\mathfrak{H}$. Hence, $\mathfrak{A}$ must be provable in $\mathbf{L P}$.

Thus $E$ is proved to be an embedding of $\operatorname{LP}(\alpha)$ into $\operatorname{LO}(\alpha \cup\{R\})$.

(5.2 T) Any $\mathbf{J}$-series logic is interpretably equivalent to $\mathbf{L O}$.

Provable by making use of $(1.6 \mathrm{R}),(2.10 \mathrm{R})$, and $(5.1 \mathrm{~T})$.

(5.3 T) LQ can be faithfully interpreted in LO.

Proof. Let $\mathbf{L Q}(\alpha)$ be any $\mathbf{L Q}$-system and $\alpha$ be $\left\{A_{i}, \ldots, A_{k}\right\}$. Let us take up a proposition symbol $F$ (0-ary relation symbol) lying outside of $\alpha$.

I can now define an embedding $\mathbf{E}$ of $\mathbf{L Q}(\alpha)$ in $\mathbf{L O}(\alpha \cup\{F\})$ by the following rule of associations:

$$
\mathrm{E}: \quad \rightarrow \longrightarrow \rightarrow, \quad(\mathrm{l}) \longrightarrow\left(\mathrm{)}, \wedge \longrightarrow \wedge_{F}, \quad \vee \longrightarrow \underset{F}{\vee}, \quad(\exists) \longrightarrow(\underset{F}{\exists}), \quad A_{i} \longrightarrow A_{i}^{F}\right.
$$

Let $\mathfrak{A}$ be any proposition (naturally a P-proposition) of $\mathbf{L Q}(\alpha)$, and $\mathfrak{A}[F]$ be its word-for-word translation in $\mathbf{L O}(\alpha \cup\{F\})$ by $E$. By virtue of (3.12 T), also the translation of Rule XIV holds in LO, so we can see taking ( $3.6 \mathbf{R})$, (4.3 $\mathbf{T})$, and $(4.10 \mathrm{R})$ into consideration that $\mathfrak{A}[F]$ is provable in $\operatorname{LO}(\alpha \cup\{F\})$ as far as $\mathfrak{H}$ is provable in $\mathbf{L Q}(\alpha)$.

Next, I will show that $\mathfrak{A}$ is provable in $\mathbf{L Q}(\alpha)$ as far as $\mathfrak{A}[F]$ is provable in $\operatorname{LO}(\alpha \cup\{F\})$. As $\mathbf{L O}$ is a logic weaker than $\mathbf{L K}, \mathfrak{A}[F]$ is surely provable in $\mathbf{L K}(\alpha \cup\{F\})$ as far as it is provable in $\mathbf{L O}(\alpha \cup\{F\})$.

Accordingly, the proposition $\mathfrak{X}[\wedge]$ i.e. the proposition obtained on replacing every $F$ in $\mathfrak{2}[F]$ by $\wedge$ is surely provable in $\mathbf{L K}$. Since $\mathbf{L Q}$ is a logic weaker than $\mathbf{L K}, \mathfrak{U}$ is equivalent to $\mathfrak{U}[\wedge]$ in $\mathbf{L K}$ according to $(3.6 \mathrm{R}),(3.9 \mathrm{R}),(4.11 \mathrm{R})$, and a remark in (2.1) that $A_{i}^{\wedge}$ i.e. $\left(A_{i} \rightarrow \hat{\wedge}\right) \rightarrow \wedge$ is equivalent to $A_{i}$ in LK. Hence, $\mathfrak{A}$ is provable in LK. Because $\mathfrak{A}$ is a $\mathbf{P}$-proposition of $\mathbf{L Q}(\alpha), \mathfrak{A}$ is also provable in $\mathbf{L Q}(\alpha)$. (See $(2.7 \mathbf{T})$.)

Thus, $\mathbf{E}$ is proved to be an embedding of $\mathbf{L Q}(\alpha)$ into $\mathbf{L O}(\alpha \cup\{\dot{F}\})$.

(5.4 T) Any $\mathbf{K}$-series logic can be faithfully interpreted in $\mathbf{L O}$.

Provable by making use of (1.4D), (1.6 R), (2.10 R), and (5.3 T). 


\section{REFERENCES}

[1] Curry, H. B., [1] The system LD, J. Symb. Log., 17 (1952), 35-42.

[2] Foundations of mathematical logic (1963).

[2] Gentzen, G., [1] Untersuchungen über das logische Schliessen, Math. Ztschr., 39 (1934) 176-210, 405-431.

[3] Glivenko, V., [1] Sur quelques points de la logique de M. Brouwer, Bull. Acad. Sci. Belg. (1929), 183-188.

[4] Hilbert, D. and Bernays, P., [1] Grundlagen der Mathematik, I (1934).

[5] Johansson, I., [1] Der Minimalkalkül, ein reduzierter intuitionistischer Formalismus, Compositio Math., 4 (1936), 119-136.

[6] Kuroda, S., [1] Intuitionistische Untersuchungen der formalistischen Logik, Nagoya Math. J., 2 (1951), 35-47.

[7] Lorenzen, P., [1] Einführung in die operative Logik und Mathematik (1955).

[8] Ono, K., [1] A certain kind of formal theories, Nagoya Math J., 25 (1965), 59-86.

[2] Logische Untersuchungen über die Grundlagen der Mathematik, J. of Fac. of Sci., Imp. Univ. of Tokyo, Sect. 1, vol. III, part 7 (1938), 329-389.

[3] On a practical way of describing formal deductions, Nagoya Math. J., 20 (1962), 115-121.

[9] Peirce, C. S., [1] On the algebra of logic; A contribution to the philosophy of notation., Amer. J. of Math., 7 (1885), 180-202.

\section{Mathematical Institute,}

Nagoya University 